\title{
PERSPECTIVE
}

\section{UV-induced damage to DNA: effect of cytosine methylation on pyrimidine dimerization}

\author{
Lara Martinez-Fernandez ${ }^{1}$, Akos Banyasz ${ }^{2}$, Luciana Esposito ${ }^{1}$, Dimitra Markovitsi ${ }^{2}$ and Roberto Improta $^{1}$
}

Methylation/demethylation of cytosine plays an important role in epigenetic signaling, the reversibility of epigenetic modifications offering important opportunities for targeted therapies. Actually, methylated sites have been correlated with mutational hotspots detected in skin cancers. The present brief review discusses the physicochemical parameters underlying the specific ultravioletinduced reactivity of methylated cytosine. It focuses on dimerization reactions giving rise to cyclobutane pyrimidine dimers and pyrimidine (6-4) pyrimidone adducts. According to recent studies, four conformational and electronic factors that are affected by cytosine methylation may control these reactions: the red-shift of the absorption spectrum, the lengthening of the excited state lifetime, changes in the sugar puckering modifying the stacking between reactive pyrimidines and an increase in the rigidity of duplexes favoring excitation energy transfer toward methylated pyrimidines.

Signal Transduction and Targeted Therapy (2017) 2, 17021; doi:10.1038/sigtrans.2017.21; published online 9 June 2017

\section{INTRODUCTION}

Methylation occurring at the 5 position of cytosine (C5-methylation; Figure 1) plays a key role in epigenetic mechanisms involved in the regulation of a variety of biological processes ranging from cell differentiation to gene expression. DNA methylation is also one of the most extensively studied epigenetic modifications in cancer. ${ }^{1}$ As the degree of methylation varies during the cell cycle, 5 -methylcytosine $(5 \mathrm{mC})$ is considered as the fifth 'dynamic' letter of the genetic code. ${ }^{2-4}$ The functions involving $5 \mathrm{mC}^{5}$ and the associated signal transduction, may be highly perturbed by chemical alteration of cytosines provoked by ultraviolet (UV) radiation. In contrast to genetic changes, epigenetic modifications are frequently reversible, which provides opportunities for targeted treatment using specific inhibitors. In this respect, the specificity of $5 \mathrm{mC}$ lies in the fact that, although it represents at most $5 \%$ of the bases in the human genome, it has been described as an endogenous mutagen. ${ }^{6,7} \mathrm{CpG}$ sites contribute to $\sim 35 \%$ of all point mutations in the germline ${ }^{8}$ and are important hotspots for acquired somatic mutations leading to cancer. ${ }^{6-13}$ The degree of correlation between methylation and cancer depends on the tumor type. ${ }^{6}$ In particular, recent studies show that $\sim 40 \%$ of melanomas are connected with C5-methylation. ${ }^{12}$ It is well known that $5 \mathrm{mC}$ is a mutable site, involved in inherited diseases and in tumors, because it can undergo spontaneous deamination to thymine. ${ }^{14,15}$ Concerning UV-induced mutations, that is the focus of the present contribution, methylation has been correlated with the formation of cyclobutane pyrimidine dimers (CPDs) (Figure 1) occurring in X5mCG sequences, where $X$ represents a thymine $(\mathrm{T})$ or a cytosine $(\mathrm{C}){ }^{6,16-18}$

In a more general way, CPDs may be formed via a direct or an indirect mechanism. ${ }^{19}$ The former involves absorption of photons directly by DNA, while in the latter photons are absorbed by other molecules present in cell, which subsequently react with the nucleic acids. The indirect mechanism is context dependent, being affected by factors such as metabolism, pollution or drugs.
In contrast, the direct mechanism corresponds to an intrinsic property of DNA and deserves particular attention.

UV-induced reactions establishing chemical bonds between neighboring pyrimidines lead mainly to two families of dimers (Figure 1): cyclobutane pyrimidine dimers, which may be formed as cis-syn (c,s CPDs) and trans-syn ( $t, s$ CPDs) stereoisomers, and pyrimidine (6-4) pyrimidone adducts (64PPs). Both types of photoproducts have been detected in dinucleoside monophosphates when one of the reacting pyrimidines is $5 \mathrm{mC} .^{20}$ The resulting CPDs may deaminate giving rise to the corresponding thymine photoproducts. ${ }^{17,20-22}$ Such secondary reactions occurring in DNA sequences in which both $\mathrm{T} 5 \mathrm{mC}$ and $\Pi \mathrm{T}$ repeats are present render the direct correlation between cytosine methylation and dimerization delicate.

A few studies, performed on model systems, genomic and cellular DNA, investigated how methylation affects reaction yields and/or induction ${ }^{20,23-25}$ and reported that it enhances CPD formation, especially for UVB irradiation. Accordingly, among the numerous studies searching the reasons for the increased UVinduced mutagenicity associated with $5 \mathrm{mC}$, one direction explores the very first steps of the complex cascade of events, intervening between photon absorption by DNA and chemical reactions. Performed in vitro or in silico, such investigations focus on the elementary processes, trying to describe the fate of the photon energy within DNA.

The purpose of the present article is to provide a critical review of various fundamental physicochemical aspects involved in the intrinsic photoreactivity of methylated DNA, exploiting some very recent contributions that combine computational methods (quantum chemistry and molecular dynamics simulations) with optical spectroscopy.

The first section is dedicated to the effect of C5-methylation on the static and dynamic properties of the $5 \mathrm{mC}$ monomer excited state, which could affect its photochemical behavior. Then, after concisely discussing the electronic grounds that control photodimerization reactions, we examine systems with increasing

${ }^{1}$ Istituto di Biostrutture e Bioimmagini, CNR, Napoli, Italy and ${ }^{2}$ LIDYL, CEA, CNRS, Université Paris-Saclay, Gif-sur-Yvette, France.

Correspondence: R Improta (robimp@unina.it)

Received 9 October 2016; revised 24 January 2017; accepted 30 March 2017 


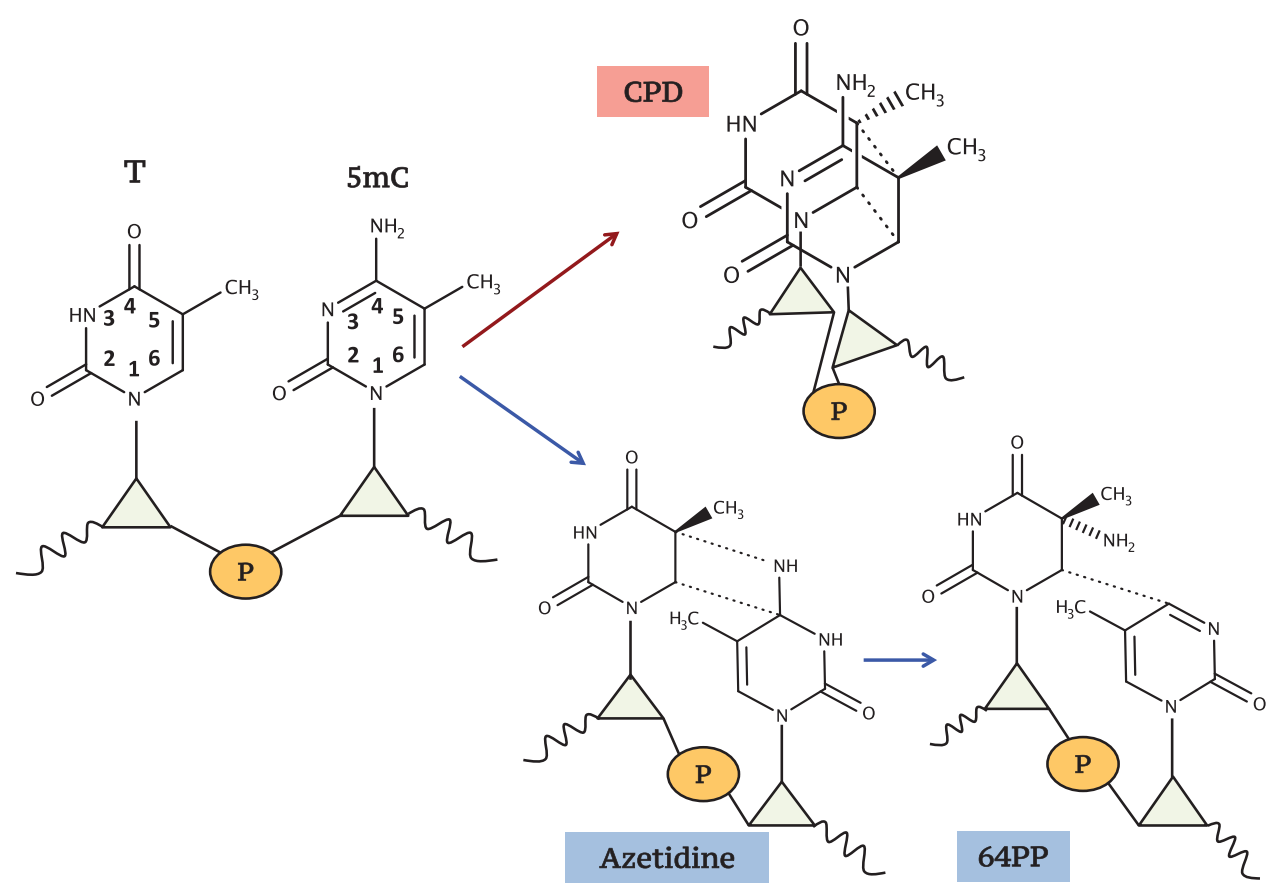

Figure 1. Formation of T5mC dimeric photoproducts. CPD, cyclobutane pyrimidine dimers (c,s stereo isomers); 64PP, pyrimidine (6-4) pyrimidone photoproducts, sugars are represented by triangles, phosphate groups by a circle.

complexity. Starting from small oligonucleotides (dinucleotides and trinucleotides) and going to longer single and double strands, we analyze how conformational and electronic modifications induced by C5-methylation influence the studied reactions. Finally, we try to provide a simple and general picture of the present knowledge on the effect of C5-methylation on the photoactivated reactivity of DNA and discuss the main perspectives in this field.

\section{MC MONOMER}

As shown in Figure 2, C5-methylation leads to a noticeable redshift $(c a .10 \mathrm{~nm})$ of the absorption maximum of the nucleosides. For both nucleosides $\mathrm{dC}$ and $5 \mathrm{mC}$, this band arises from an electronic transition with a predominant HOMO $\rightarrow$ LUMO character, that is, involving the excitation of an electron from the highest occupied molecular orbital (HOMO) to the lowest unoccupied molecular orbital (LUMO; Figure 2). This result has been rationalized by recent calculations $^{26}$ showing that the 5 -methyl substituent provides an antibonding contribution to the HOMO (as identified by the out-of-phase combination with respect to the $\mathrm{C} 5=\mathrm{C} 6 \pi$ bond), decreasing its stability and, therefore, the HOMO/LUMO gap. The red shift of the cytosine absorption spectrum upon methylation has been considered to be the cause of the increased photoreactivity of $5 \mathrm{mC}$ observed in cells. ${ }^{27}$

In addition to the spectral modification, C5-methylation also leads to a noticeable increase of the excited state lifetime. In water solutions, the lowest energy $\pi \pi^{*}$ excited state of $d C\left(S_{1}\right)$ decays mainly on the sub-ps time scale, whereas the average excited state lifetime of $5 \mathrm{mC}$ amounts to several ps. ${ }^{28-30}$ For cytosine, an almost barrierless path on $\mathrm{S}_{1}$ leads to a crossing region with the ground electronic state $\left(\mathrm{S}_{0}\right)$, giving account of the very short lifetime and of the very low fluorescence quantum yield. In contrast, in the case of the methylated analog, as discussed in detail in a forthcoming contribution, ${ }^{31}$ the potential energy surface of the lowest excited state contains a minimum, separated from the crossing region with $S_{0}$ by a sizeable energy barrier $(0.15-0.3 \mathrm{eV})$, explaining the increase of the $S_{1}$ lifetime. Since the $\mathrm{S}_{1}$ excited state is involved in the photodimerization reaction, as

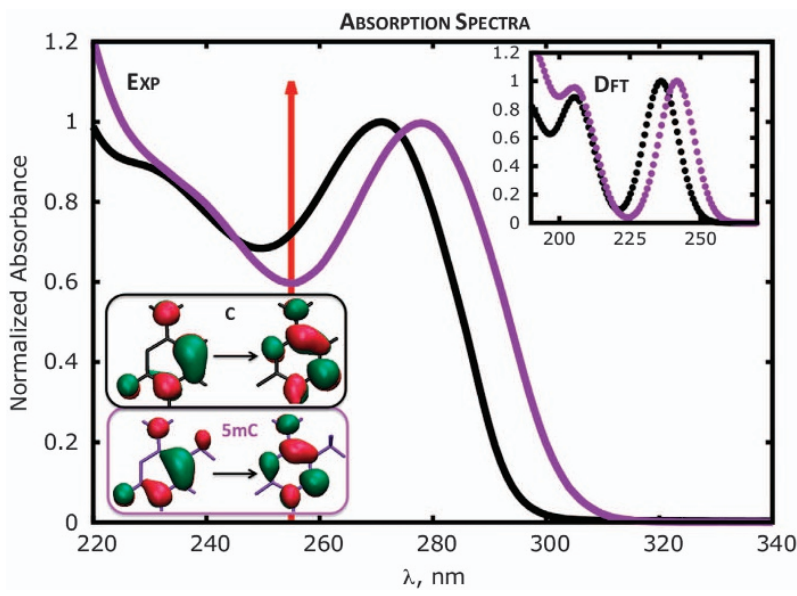

Figure 2. Effect of cytosine methylation on the absorption spectra of nucleosides in water: ${ }^{30} \mathrm{dC}$ (black) and $5 \mathrm{mC}$ (violet). The red-arrow indicates the irradiation wavelength $(255 \mathrm{~nm})$ used in the experiments providing the data reported in Tables 1 and 2. The absorbance of the lower absorption peak is set to 1. Inset: computed TDDFT absorption spectra. ${ }^{26}$

we explain below, a longer lifetime could, in principle, enhance the quantum yield of the reaction. But so far, it has not been possible to assess the significance of this effect on the ground of relevant experimental and computational studies on duplexes.

\section{PHOTODIMERIZATION: 5MC CONTAINING SHORT OLIGONUCLEOTIDES}

Before analyzing the effect of $\mathrm{C} 5$-methylation on the photodimerization quantum yields, it is useful to provide information on the electronic states involved in these reactions. For what concerns CPD formation, several studies on di-pyrimidine steps (including TC and T5mC steps) ${ }^{32-40}$ show that when two bases are stacked, their $\mathrm{S}_{1}$ excited states (the HOMO $\rightarrow$ LUMO transitions depicted in 
a

\section{CPD}

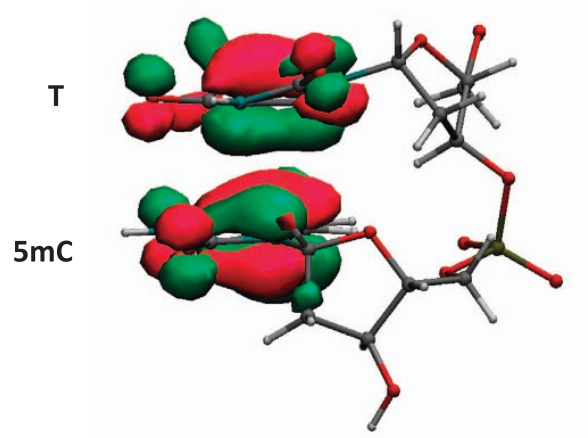

HOMO

b

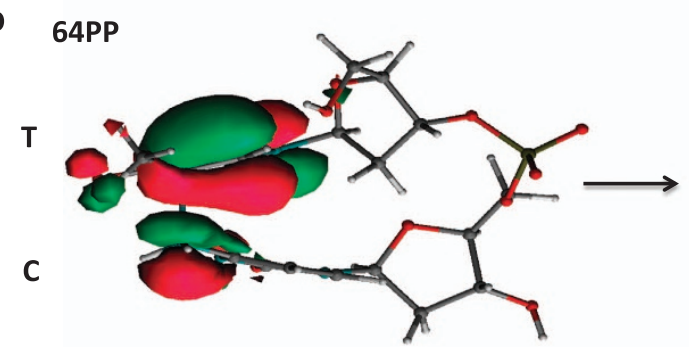

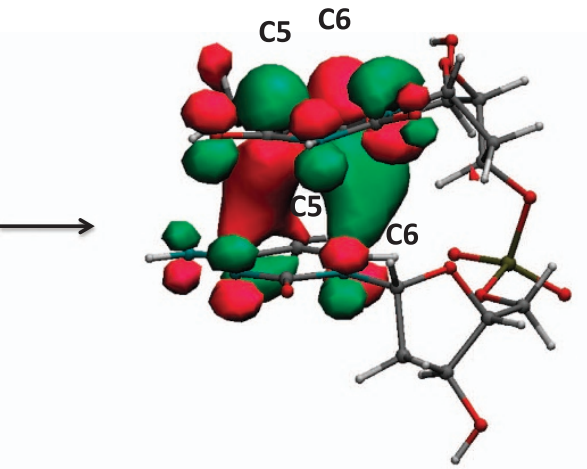

LUMO

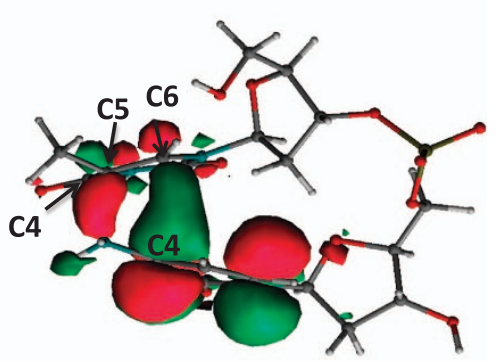

Figure 3. Frontier orbitals involved in the electronic transitions leading to CPD (a) and 64PP (b) formation.

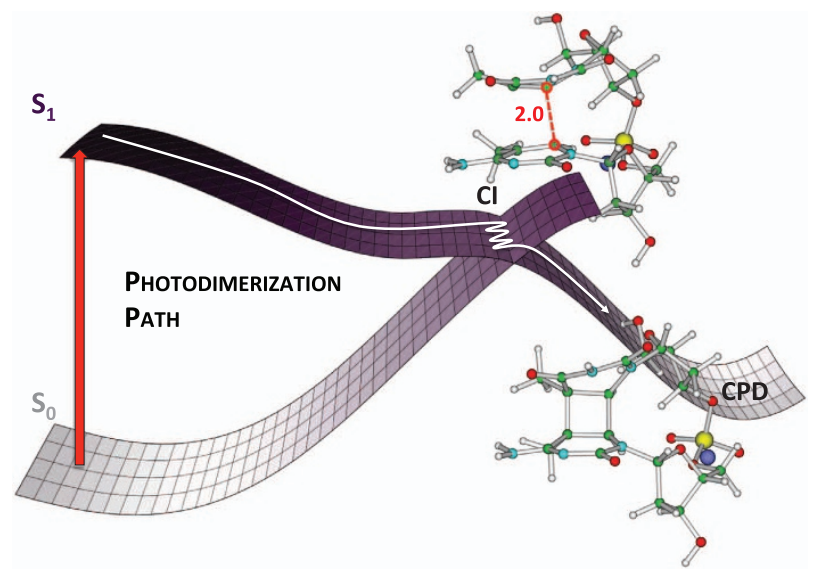

Figure 4. Schematic description of the barrierless path leading to CPD formation.

Figures 2 and 3) interact, giving rise to an electronic transition delocalized over the two pyrimidines. As shown in Figure 3a, this transition involves the excitation of an electron from a molecular orbital (MO) corresponding to the combination of the highest energy $\pi$ bonding orbitals of the two bases, to a MO deriving from the combination of the two LUMO's (exciton). Interestingly, this latter orbital has a clear bonding character between the two C5/ $\mathrm{C}^{\prime}$ and $\mathrm{C} 6 / \mathrm{C6}^{\prime}$ pairs of the two bases. Light absorption, promoting an electron to this orbital, thus makes the dimerization reaction much easier than in the ground electronic state. Actually, time resolved experimental studies showed that the CPD formation in thymine single strands is ultrafast; the two new bonds are formed essentially within $1 \mathrm{ps}$ after light absorption. ${ }^{41,42}$ In addition, according to quantum mechanical calculations, for certain stacking arrangements of the bases, CPD formation on the $S_{1}$
Table 1. Quantum yields $\phi\left(\times 10^{3}\right)$ determined for the formation of dimeric photoproducts following irradiation at $255 \mathrm{~nm}$

\begin{tabular}{lcccccc}
\hline & $T C G^{32}$ & $T 5 m C G^{32}$ & $n-s s^{50}$ & $m-s s^{50}$ & $n-d s^{50}$ & $m-d s^{50}$ \\
\hline$\phi_{\text {CPD }}(c, s)$ & 0.5 & 0.7 & 1.3 & 1.3 & 0.6 & 1.1 \\
$\phi_{64}$ & 0.6 & 0.4 & 1.4 & 0.3 & 0.7 & 0.1 \\
\hline Abbreviations: CPD, cyclobutane pyrimidine dimer; & 64, pyrimidine (6-4) \\
pyrimidone adducts; ds, double strand; m, methylated; & n, non-methylated; \\
ss, single strand.
\end{tabular}

potential energy surface (PES) is barrierless (Figure 4). ${ }^{32-40,43}$ Consequently, this photodimerization reaction is mainly governed by the ground state conformation: the couple of bases that are in a suitable conformation undergo ultrafast CPD reaction. In particular, it has been proposed that the photo-dimerization is governed by the relative frequency of structures exhibiting short distances between the reactive bonds. ${ }^{44-46}$ More recent contributions also highlight the importance of the sugar puckering adopted by each reactive nucleotide, ${ }^{32,34}$ which affects the stacking geometry of the dipyrimidine steps. Thus, it appears that several conformational parameters are important for CPD formation.

The 64PP formation has been investigated less thoroughly compared to CPDs and mostly for $\Pi$ steps. There are indications that it proceeds through the so-called oxetane intermediate ${ }^{47}$ followed by 64PP formation on the ms time-scale. ${ }^{48}$ Experiments showed that oxetane formation is faster than $200 \mathrm{~ns}^{48}$ but the precise dynamics of the reaction has not been characterized so far. QM studies suggest that the oxetane in the case of TT steps or the azetidine for TC steps are formed on the PES of an electronic state with charge transfer character. ${ }^{32,33}$ The latter can be described as arising from the transfer of an electron from the HOMO of the pyrimidine on the $5^{\prime}$-end toward the LUMO of that on the $3^{\prime}$-end (Figure $3 b)^{33}$ The presence of an energy barrier either in the step 
Table 2. Quantification of the effect of C5-methylation on the pyrimidine dimerization following irradiation at $255 \mathrm{~nm}$ by the parameter $I_{\mathrm{m}}=\phi_{\mathrm{m}} \psi_{\mathrm{n}} / \phi_{\mathrm{n}} \psi_{\mathrm{m}}$ accounting for the different absorption spectra of $\mathrm{C}$ and $5 \mathrm{mC} ; \phi_{\mathrm{m}}$ and $\phi_{\mathrm{n}}$ are the quantum yields found for the methylated and the corresponding non-methylated system, respectively (Table 1 ); $\psi_{\mathrm{m}}$ and $\psi_{\mathrm{n}}$ represent the fraction of photons absorbed by a reactive $5 \mathrm{mC}$ or $\mathrm{C}$

\begin{tabular}{lccc}
\hline & Trinucleo tides $^{32}$ & Single strands $^{50}$ & Double strands $^{50}$ \\
\hline CPDs $(c, s)$ & 1.7 & 1.3 & 2.4 \\
64PPs & 0.8 & 0.3 & 0.3 \\
\hline
\end{tabular}

Abbreviations: CPD, cyclobutane pyrimidine dimer; 64, pyrimidine (6-4) pyrimidone adducts.

leading to the oxetane formation, ${ }^{33}$ or in that leading to the final photoproduct can explain the smaller yield of 64 PP than CPD. ${ }^{49}$

Coming to the experimental results on pyrimidine photodimerization in short oligomers, the first study, performed on 1994 for $5 \mathrm{mC}$-containing dinucleoside monophosphates, ${ }^{20}$ noticed that $\mathrm{C}_{5}$ methylation enhances CPD formation. Two decades later, dimerization quantum yields $\phi$ (and not simple yields), which are necessary for the assessment of the intrinsic reactivity of $5 \mathrm{mC}$, were reported for trinucleotides containing the biologically relevant sequence $\mathrm{T} 5 \mathrm{mC}$ and compared to those observed for the non-methylated analogs TCG. ${ }^{32}$ As in the case of the early study, trinucleotides were irradiated at $255 \mathrm{~nm}$. The quantum yields determined for both CPDs $\left(\phi_{\mathrm{CPD}}\right)$ and 64PPs $\left(\phi_{64}\right)$ are shown in Table 1. In line with what was found for dinucleotides, a higher $\phi_{\text {CPD }}$ was determined for T5mCG compared to TCG.

According to its definition, the quantum yield equals to the number of formed dimers divided by the number of absorbed photons. Thus, in order to check to what extent the observed variations of quantum yields arise from the modification of the absorption spectrum of cytosine upon methylation, its contribution can be quantified by the following new parameter: ${ }^{32}$ $I_{\mathrm{m}}=\phi_{\mathrm{m}} \psi_{\mathrm{n}} / \phi_{\mathrm{n}} \psi_{\mathrm{m}}$, where $\phi_{\mathrm{m}}$ and $\phi_{\mathrm{n}}$ are the quantum yields found for the methylated and the corresponding non-methylated system, respectively (Table 2). We remark that the $I_{\mathrm{m}}$ values determined for C,S CPDs and 64PPs, 1.7 and 0.8, respectively, being significantly different than 1 , reveal that other factors independent from the photon absorption, affect also the pyrimidine dimerization. It is worth-noticing that in contrast to C5-methylation, N4-methylation of cytosine in trinucleotides enhances the yield of 64PPs. ${ }^{51}$

Computational analysis, combining quantum mechanical calculations on $\mathrm{TC} / \mathrm{T} 5 \mathrm{mC}$ dinucleotides and molecular dynamics simulations on TCG/T5mCG trinucleotides, shows indeed that C5-methylation induces weak, but noticeable structural effects, modulating the conformational equilibria of the dipyrimidine steps. For example, the $\mathrm{C2}^{\prime}$ endo-C1'exo (c2c1) conformer is more stable than the $\mathrm{C2}^{\prime}$ endo- $\mathrm{C2}^{\prime}$ endo $(\mathrm{c} 2 \mathrm{c} 2)$ one for $\mathrm{T} 5 \mathrm{mC}$, while the opposite is found for $\mathrm{TC}^{32}$ In general, C5-methylation induces a decrease of the pseudo-rotation phase angle, measuring the sugar ring puckering. ${ }^{32}$ Excited state QM calculations show that, independently of C5-methylation, c2c2 conformations are not reactive, since the favored decay path involves localization of the excitation on a single base, followed by ultrafast decay to $\mathrm{S}_{0}$ (monomer like decay pathway). On the opposite, for c2c1 arrangement a barrierless path on $\mathrm{S}_{1}$ leads to CPD formation. Shortly, C5-methylation favors puckering combinations and, thus, stacking arrangements, that are favorable to CPD formation but less prone to 64PP formation. The role of C5-methylation in modulating the relative importance of 'monomer like' and 'dimerization' paths has been highlighted in QM studies of other dipyrimidine sequences. ${ }^{40}$

\section{LONGER SEQUENCES CONTAINING $5 \mathrm{MC}$}

Mitchel determined the induction time of CPDs and 64PPs in various model duplexes and concluded that C5-methylation enhances the formation of both types of dimers. ${ }^{23}$ However, it is not possible to correlate the induction time, which is a phenomenological parameter, with physico-chemical factors underlying the reaction mechanisms. Moreover, the conclusions regarding the 64PP enhancement contrasts with an in vivo study. ${ }^{52}$

Only one study reports the effect of C5-methylation on the CPD yields in naked human genomic DNA in solution. ${ }^{24}$ Performed in vitro, under conditions where solely the direct mechanism is operative and discriminating clearly dimers arising from methylated/non-methylated cytosines, this investigation revealed an interesting point: upon $254 \mathrm{~nm}$ irradiation the same CPD yield was observed for methylated and non-methylated DNA, although at this wavelength the molar absorption coefficient of dC is $30 \%$ higher than that $5 \mathrm{mC}$.

The above point was further investigated using suitably tailored oligonucleotides: single strands $(\text { T5 } 5 \text { CGTA })_{3}$ and $(\text { TCGTA })_{3}$ and duplexes $(\text { T5mCGTA) })_{3} \cdot(\text { TACGA })_{3}$ and $(\text { TCGTA })_{3} \cdot(\text { TACGA })_{3} .{ }^{50}$ Their sequence was chosen in a way that pyrimidine dimers arise only from sites that can be methylated so that to avoid confusion with dimers arising from $\Pi$ sites. The most striking finding is that the $I_{\mathrm{m}}$ (Table 2) value (representing the change in the reactivity of cytosine upon methylation) determined for duplexes is higher at the shorter wavelength; it is 2.4 at $255 \mathrm{~nm}$ and only 1.3 at $282 \mathrm{~nm}$. In contrast, in the case of single strands, although the irradiation wavelength affects $\phi_{\mathrm{CPD}}$, it does not have a noticeable effect on $I_{\mathrm{m}}$. Thus, the observation on duplexes demonstrate, in a more pronounced way than for short systems and longer single strands, that several other factors are responsible for the modification of dimerization efficiency following C5-methylation. From the conformational point of view, molecular dynamics simulations have been applied to DNA fragments with a different extent of methylation (either fully methylated or hemimethylated) and with different sequences (either with repetitive CG sequences, or segments containing interdispersed CG sequences), ${ }^{53-57}$ focusing mainly on CG steps. All these studies agree that C5-methylation decreases the DNA flexibility.

For what concerns the TCG containing long sequences, whose photodimerization quantum yields and $I_{\mathrm{m}}$ values are also reported in Tables 1 and 2, respectively, MD simulations have shown that the trends found in trinucleotides are maintained in longer singlestranded stretches. ${ }^{32,50}$ In particular, C5-methylation destabilizes the stacking of CG step, favors cytidine C1'exo versus C2'endo conformers, giving rise to a larger population of molecules with short distances between reactive bonds involved in CPD formation. On the other hand, though the average structural features of duplexes are less impacted by C5-methylation, the amplitude of conformational motions is significantly smaller in methylated structures, confirming the results obtained on other sequences. ${ }^{53-57}$ In particular, the increased rigidity of the $5 \mathrm{mC}$ containing duplexes is also revealed by the lower s.d. of the distribution of the distances between the 'photo-reactive' bonds. ${ }^{32}$

Quantum mechanical calculations on fragments of these duplexes show that the UV absorption populates excited states delocalized over two or more bases. ${ }^{50}$ The electronic transitions contributing to the maximum of the experimental absorption peak (around $260 \mathrm{~nm}$ ) are significantly coupled with potentially photochemically active excited states, delocalized over bispyrimidine steps. There are thus hints that energy transfer takes place; internal conversion among exciton states (intraband scattering) leads to the bottom of the exciton band, as found experimentally for several nonmethylated DNA duplexes and G-quadruplexes. ${ }^{58,59} \mathrm{C} 5$-methylation can enhance the efficiency of 


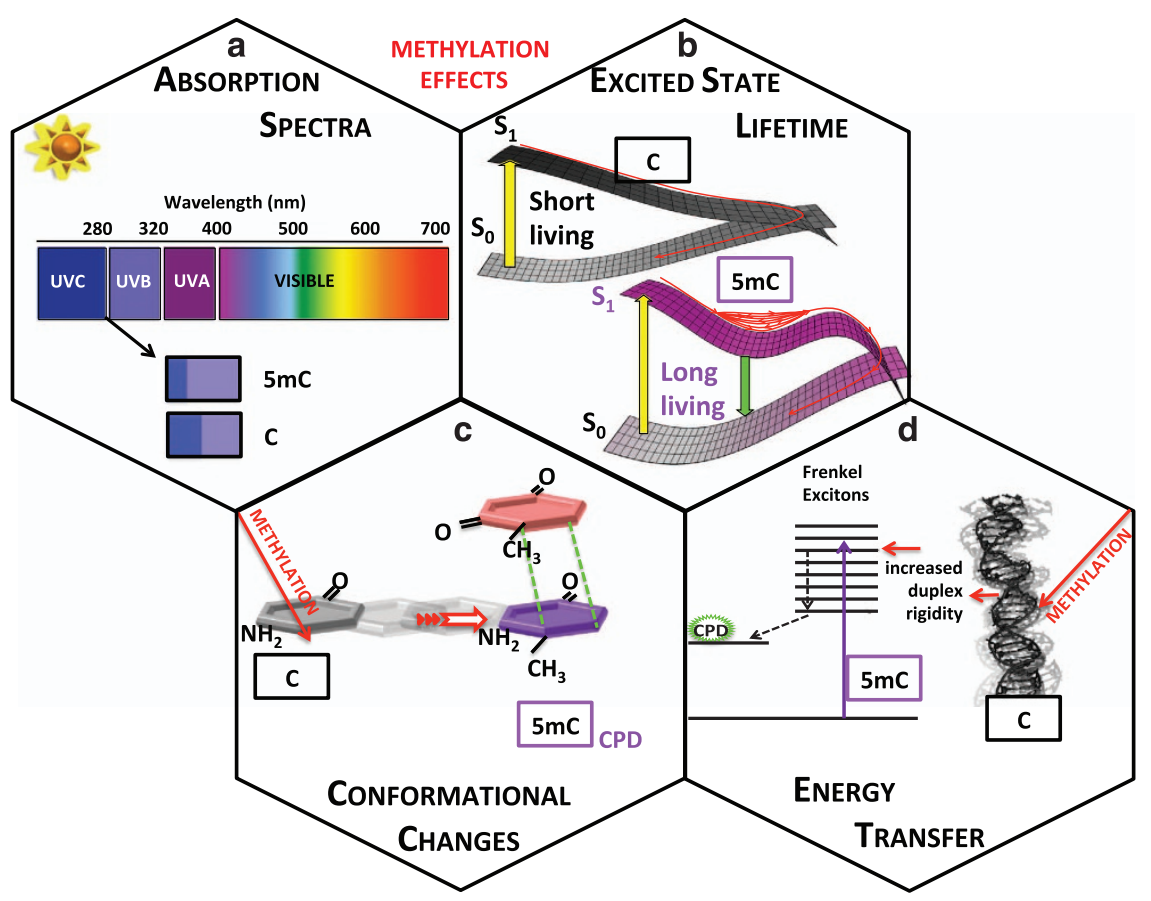

Figure 5. Synoptic scheme summarizing the most significant factors accounting for the effect for C5-methylation on pyrimidine dimerization.

intraband scattering, either by increasing the rigidity of the duplex structure (as discussed above), or by decreasing the energy of the lowest energy excited states in reactive dipyrimidine steps.

\section{CONCLUDING REMARKS AND PERSPECTIVES}

In this contribution, we have provided a general picture regarding the effect of C5-methylation on pyrimidine dimerization and discussed the various physico-chemical factors that may underlie this effect. These factors are schematically illustrated in Figure 5. It is clear that the experimentally observed effects cannot be explained by a single cause and that a subtle interplay among various factors governs the reactivity of methylated DNA.

The red shift of the absorption spectrum induced by C5-methylation (Figure 5a), due to energy destabilization of the HOMO orbital, is certainly an important factor intervening in both dimerization reactions, but it is not the only factor into play. The lengthening of the excited state lifetime (Figure 5b) could contribute to CPD enhancement, but so far there are no available experimental results on duplexes supporting this point. For all types of methylated systems, structural effects, favoring conformations more reactive toward CPD formation (Figure $5 \mathrm{c}$ ) and modulating the role of the flanking bases, ${ }^{60,61}$ are certainly a key factor. Finally, C5-methylation increases the duplex rigidity (Figure 5d), facilitating the energy transfer from non-reactive bases to reactive ones, via delocalized excited states (excitons).

The role of the above-mentioned factors is more easily assessed in the case of CPDs, whose formation takes place in a single step via delocalized excited states.

As 64PP formation takes place via a two-step mechanism (the first involving a charge transfer excited state between the two reactive pyrimidines) and the experimental observations concern so far the overall reaction, the direct correlation of calculated parameters with experimental observations is delicate.

Notwithstanding the significant advances made since the beginning of the 21st century in our understanding of how DNA methylation affects pyrimidine dimerization caused by direct absorption of UV radiation, several important issues remain to be elucidated.

Starting from photon absorption, it would be interesting to explore the absorption of methylated systems in the UVA spectral domain. In the case of non-methylated systems, it was shown recently that, in contrast to the isolated bases, both naked genomic DNA and model duplexes absorb in this spectral domain. This absorption, giving rise to CPDs, ${ }^{62,63}$ was correlated to charge transfer transitions among reactive pyrimidines, ${ }^{34}$ which may be vibronically coupled to $\pi \pi^{*}$ transitions.

Regarding the role of the excited state lifetime, ultrafast spectroscopy, probing the IR spectral domain, already used for the study of thymine CPDs, could bring valuable information for the dynamics of dimerization reactions.

The conclusions concerning the role of conformation and energy transfer were drawn from joint experimental and theoretical investigations on systems containing the sequence TCG/T5mCG. Similar studies on other sequences, as for example CCG/C5mCG, would allow checking in which extent these conclusions have a general validity.

\section{COMPETING INTEREST}

The authors declare no conflict of interest.

\section{REFERENCES}

1 Baylin SB, Jones PA. A decade of exploring the cancer epigenome-biological and translational implications. Nat Rev Cancer 2011; 11: 726-734.

2 Holliday R, Pugh JE. DNA modification mechanisms and gene activity during development. Science 1975; 187: 226.

3 Lister R, Ecker JR. Finding the fifth base: genome-wide sequencing of cytosine methylation. Genome Res 2009; 19: 959-966.

4 Smith ZD, Meissner A. DNA methylation: roles in mammalian development. Nat Rev Genet 2013; 14: 204-220.

5 Jones PA. Functions of DNA methylation: islands, start sites, gene bodies and beyond. Nat Rev Genet 2012; 13: 484-492. 
6 Robertson KD, Jones PA. DNA methylation: past, present and future directions. Carcinogenesis 2000; 21: 461-467.

7 Rideout WM, Coetzee GA, Olumi AF, Jones PA. 5-Methylcytosine as an endogenous mutagen in the human LDL receptor and p53 genes. Science 1990; 249: 1288.

8 Cooper DN, Youssoufian H. The CpG dinucleotide and human genetic disease. Hum Genet 1988; 78: 151-155.

9 Greenblatt MS, Bennett WP, Hollstein M, Harris CC. Mutations in the p53 tumor suppressor gene: clues to cancer etiology and molecular pathogenesis. Cancer Res 1994; 54: 4855-4878.

10 Denissenko MF, Chen JX, Tang M-S, Pfeifer GP. Cytosine methylation determines hot spots of DNA damage in the human P53 gene. Proc Natl Acad Sci USA 1997; 94: 3893-3898.

11 Pfeifer G, You Y, Besaratinia A. Mutations induced by ultraviolet light. Mut Res 2005; 571: 19-31.

12 Jin SG, Xiong WY, Wu XW, Yang L, Pfeifer GP. The DNA methylation landscape of human melanoma. Genomics 2015; 106: 322-330.

13 Schulz W, Seifert H-H. DNA Methylation and Cancer Therapy (Medical Intelligence Unit), vol. 2005. Springer: New York, 2005, pp 42-58.

14 Holliday R, Grigg GW. DNA methylation and mutation. Mutat Res 1993; 285: 61-67.

15 Cooper DN, Mort M, Stenson PD, Ball EV, Chuzhanova NA. Methylation-mediated deamination of 5-methylcytosine appears to give rise to mutations causing human inherited disease in $\mathrm{CpNpG}$ trinucleotides, as well as in $\mathrm{CpG}$ dinucleotides. Hum Genomics 2010; 4: 406-410.

16 Drouin R, Therrien JP. UVB-induced cyclobutane pyrimidine dimer frequency correlates with skin cancer mutational hotspots in p53. Photochem Photobiol 1997; 66: 719-726.

17 Cannistraro VJ, Taylor JS. Acceleration of 5-methylcytosine deamination in cyclobutane dimers by $\mathrm{G}$ and its implications for UV-induced C-to-T mutation hotspots. J Mol Biol 2009; 392: 1145-1157.

18 Ikehata H, Ono T. The mechanisms of UV mutagenesis. J Radiat Res 2011; 52: $115-125$

19 Cadet J, Grand A, Douki T. Solar UV radiation-induced DNA bipyrimidine photoproducts: formation and mechanistic insights. Top Curr Chem 2015; 356: 249-275.

20 Douki T, Cadet J. Formation of cyclobutane dimers and (6-4) photoproducts upon far-UV photolysis of 5-methylcytosine-containing dinucleoside monophosphates. Biochemistry 1994; 33: 11942-11950.

21 Shetlar MD, Basus VJ, Falick AM, Mujeeb A. The cyclobutane dimers of 5-methylcytosine and their deamination products. Photochem Photobiol Sci 2004; 3: $968-979$.

22 Celewicz L, Mayer M, Shetlar MD. The photochemistry of thymidylyl-(3'-5')-5methyl-2'-deoxycytidine in aqueous solution. Photochem Photobiol 2005; 81: 404-418.

23 Mitchel DL. Effects of cytosine methylation on pyrimidine dimer formation in DNA. Photochem Photobiol 2000; 71: 162-165.

24 Rochette PJ, Lacoste S, Therrien JP, Bastien N, Brash DE, Drouin R. Influence of cytosine methylation on ultraviolet-induced cyclobutane pyrimidine dimer formation in genomic DNA. Mutat Res 2009; 665: 7-13.

25 Tommasi S, Denissenko MF, Pfeifer GP. Sunlight induces pyrimidine dimers preferentially at 5-methylcytosine bases. Cancer Res 1997; 57: 4727-4730.

26 Martínez-Fernández L, Pepino AJ, Segarra-Martí J, Banyasz A, Garavelli M, Improta R. Computing the absorption and emission spectra of 5-methylcytidine in different solvents: a test-case for different solvation models. J Chem Theor Comput 2016; 12: 4430-4439.

27 You Y-H, Pfeifer GP. Similarities in sunlight-induced mutational spectra of CpG-methylated transgenes and the P53 gene in skin cancer point to an important role of 5-methycytosine residues in solar UV mutagenesis. $J \mathrm{Mol}$ Biol 2001; 305: 389-399.

$28 \mathrm{Ma}$ C, Cheng CC-W, Chan CT-L, Chan RC-T, Kwok W-M. Remarkable effects of solvent and substitution on the photo-dynamics of cytosine: a femtosecond broadband time-resolved fluorescence and transient absorption study. Phys Chem Chem Phys 2015; 17: 19045-19057.

29 Malone RJ, Miller AM, Kohler B. Singlet excited-state lifetimes of cytosine derivatives measured by femtosecond transient absorption. Photochem Photobiol 2003; 77: 158-164.

30 Sharonov A, Gustavsson T, Marguet S, Markovitsi D. Photophysical properties of 5-methylcytidine. Photochem Photobiol Sci 2003; 2: 362-364.

31 Martínez-Fernández L, Pepino AJ, Segarra-Martí J, Jovaišaitė J, Vaya I, Nenov A et al. The Photophysics of Deoxycytidine and 5-methyl-deoxycytidine in Solution: a comprehensive picture by Quantum Mechanical Calculations and Femtosecond Fluorescence Spectroscopy (submitted)

32 Esposito L, Banyasz A, Douki T, Perron M, Markovitsi D, Improta R. Effect of C5-methylation of cytosine on the photoreactivity of DNA: a Joint Experimental and Computational Study of TCG trinucleotides. J Am Chem Soc 2014; 136: 10838-10841.

33 Banyasz A, Douki T, Improta R, Gustavsson T, Onidas D, Vayá I et al. Electronic excited states responsible for dimer formation upon UV absorption directly by thymine strands: Joint Experimental and Theoretical Study. J Am Chem Soc 2012; 134: 14834-14845.

34 Improta R. Photophysics and photochemistry of thymine deoxy-dinucleotide in water: a PCM/TD-DFT Quantum Mechanical Study. J Phys Chem B 2012; 116: 14261-14274.

35 Improta R, Santoro F, Blancafort L. Quantum mechanical studies on the photophysics and the photochemistry of nucleic acids and nucleobases. Chem Rev 2016; 116: 3540-3593.

36 Spata VA, Lee W, Matsika S. Excimers and exciplexes in photoinitiated processes of oligonucleotides. J Phys Chem Lett 2016; 7: 976-984.

37 Blancafort L, Migani A. Modeling thymine photodimerizations in DNA: mechanism and correlation diagrams. J Am Chem Soc 2007; 129: 14540-14541.

38 Boggio-Pasqua M, Groenhof G, Schäfer LV, Grubmüller H, Robb MA. Ultrafast deactivation channel for thymine dimerization. J Am Chem Soc 2007; 129: 10996-10997.

39 Roca-Sanjuan D, Olaso-Gonzalez G, Gonzalez-Ramirez I, Serrano-Andres L, Merchan M. Molecular basis of DNA photodimerization: Intrinsic production of cyclobutane cytosine dimers. J Am Chem Soc 2008; 130: 10768-10779.

40 González-Ramírez I, Roca-Sanjuán D, Climent T, Serrano-Pérez JJ, Merchán M, Serrano-Andrés $L$ et al. On the photoproduction of DNA/RNA cyclobutane pyrimidine dimers. Theor Chem Acc 2011; 128: 705-711.

41 Schreier WJ, Schrader TE, Koller FO, Gilch P, Crespo-Hernández CE, Swaminathan VN et al. Thymine dimerization in DNA is an ultrafast photoreaction. Science 2007; 315: 625-629.

42 Schreier WJ, Kubon J, Regner N, Haiser K, Schrader TE, Zinth W et al. Thymine dimerization in DNA model systems: cyclobutane photolesion Is predominantly formed via the singlet channel. J Am Chem Soc 2009; 131: 5038-5039.

43 Schreier WJ, Gilch P, Zinth W. Early events of DNA photodamage. Annu Rev Phys Chem 2015; 66: 497-519.

44 Johnson AT, Wiest O. Structure and dynamics of poly(T) single-strand DNA: implications toward CPD formation. J Phys Chem B 2007; 111: 14398-14404.

45 Law YK, Azadi J, Crespo-Hernandez CE, Olmon E, Kohler B. Predicting thymine dimerization yields from molecular dynamics simulations. Biophys $J$ 2008; 94: 3590-3600.

46 McCullagh M, Lewis F, Markovitsi D, Douki T, Schatz GC. Conformational control of TT dimerization in DNA conjugates. A molecular dynamics study. J Phys Chem B 2010; 114: 5215-5221.

47 Varghese AJ, Wang SY. Thymine-thymine adduct as a photoproduct of thymine. Science 1968; 160: 186-187.

48 Marguet S, Markovitsi D. Time-resolved study of thymine dimer formation. J Am Chem Soc 2005; 127: 5780-5781.

49 Labet V, Jorge N, Morell C, Douki T, Grand A, Cadet J et al. UV-induced formation of the thymine-thymine pyrimidine (6-4) pyrimidone photoproduct-a DFT study of the oxetane intermediate ring opening. Photochem Photobiol Sci 2013; 12: 1509-1516.

50 Banyasz A, Esposito L, Douki T, Perron M, Lepori C, Improta R et al. Effect of C5-methylation of cytosine on the UV-induced reactivity of duplex DNA-conformational and electronic factors. J Phys Chem B 2016; 120: 4232-4242.

51 Douki T, Meador JA, Berard I, Wack A. N4-methylation of cytosine drastically favors the formation of (6-4) photoproducts in a TCG context. Photochem Photobiol 2015; 91: 102-108.

52 Pfeifer GP, Drouin R, Riggs AD, Holmquist GP. In vivo mapping of a DNA adduct at nucleotide resolution: detection of pyrimidine (6-4) pyrimidone photoproducts by ligation-mediated polymerase chain reaction. Proc Natl Acad Sci USA 1991; 88: 1374-1378.

53 Pérez A, Castellazzi CL, Battistini F, Collinet K, Flores O, Deniz O et al. Impact of methylation on the physical properties of DNA. Biophys $J$ 2012; 102: 2140-2148.

54 Temiz NA, Donohue DE, Bacolla A, Luke BT, Collins JR. The role of methylation in the intrinsic dynamics of B- and Z-DNA. PLoS One 2012; 7: e35558.

55 Rauch C, Trieb M, Wellenzohn B, Loferer M, Voegele A, Wibowo FR et al. C5methylation of cytosine in B-DNA thermodynamically and kinetically stabilizes $\mathrm{Bl}$. J Am Chem Soc 2003; 125: 14990-14991.

56 Derreumaux S, Chaoui M, Tevanian G, Fermandjian S. Impact of CpG methylation on structure, dynamics and solvation of cAMP DNA responsive element. Nucleic Acids Res 2001: 29: 2314-2326.

57 Ngo TTM, Yoo J, Dai Q, Zhang Q, He C, Aksimentiev A et al. Effects of cytosine modifications on DNA flexibility and nucleosome mechanical stability. Nat Commun 2016; 7: 10813. 
58 Markovitsi D. UV-induced DNA damage: the role of electronic excited states. Photochem Photobiol 2016; 92: 45-51.

59 Changenet-Barret $\mathrm{P}$, Hua $\mathrm{Y}$, Markovitsi D. Electronic excitations in guanine quadruplexes. Top Curr Chem 2015; 356: 183-202.

60 Pan ZZ, Hariharan M, Arkin JD, Jalilov AS, McCullagh M, Schatz GC et al. Electron donor-acceptor interactions with flanking purines influence the efficiency of thymine photodimerization. I Am Chem Soc 2011; 133: 20793-20798.

61 Bucher DB, Kufner CL, Schlueter A, Carell T, Zinth W. UV-induced charge transfer states in DNA promote sequence selective self-repair. J Am Chem Soc 2016; 138: 186-190.

62 Banyasz A, Vayá I, Changenet-Barret P, Gustavsson T, Douki T, Markovitsi D. Base-pairing enhances fluorescence and favors cyclobutane dimer formation induced upon absorption of UVA radiation by DNA. J Am Chem Soc 2011; 133: 5163-5165.
63 Mouret S, Philippe C, Gracia-Chantegrel J, Banyasz A, Karpati S, Markovitsi D et al. UVA-induced cyclobutane pyrimidine dimers in DNA: a direct photochemical mechanism? Org Biomol Chem 2010; 8: 1706-1711.

(i) This work is licensed under a Creative Commons Attribution 4.0 International License. The images or other third party material in this article are included in the article's Creative Commons license, unless indicated otherwise in the credit line; if the material is not included under the Creative Commons license, users will need to obtain permission from the license holder to reproduce the material. To view a copy of this license, visit http://creativecommons.org/licenses/ by/4.0/

(c) The Author(s) 2017 